IMA Journal of Applied Mathematics (2017) Page 1 of 17 doi:10.1093/imamat/xxx000

\title{
Solitary gravity waves and free surface flows past a point vortex
}

\author{
ALEX DOAK* \\ University College London \\ *alexander.doak.11@ucl.ac.uk \\ JEAN-MARC VANDEN-BRoECK \\ University College London
}

[Received on 20 July 2017]

\begin{abstract}
Nonlinear free surface flows past a disturbance in a channel of finite depth are considered. The fluid is assumed to be incompressible and inviscid and the flow to be two-dimensional, irrotational and supercritical. The disturbance is chosen to be a point vortex. Highly accurate numerical solutions are computed. The basic idea of the numerical approach is first to develop codes to compute solitary waves and then to introduce appropriate modifications to model the point vortex. Previous results are recovered and new solutions are presented.
\end{abstract}

Keywords: solitary waves; free surface flows; point vortex.

\section{Introduction}

This paper is concerned with two related potential free surface flows problems. The effect of gravity is included in the dynamic boundary condition but the effect of surface tension is neglected. In both problems the flows are assumed to be two-dimensional and steady.

The first problem is the computation of solitary waves. Many papers have been written on the subject (see Schwartz \& Fenton (1982) for a review and Vanden-Broeck (2010) for more recent results). Solitary waves are non-periodic waves characterised by a flat free surface in the far field. They can be calculated by assuming a steady flow in a frame of reference moving with the wave. For small amplitudes, solitary waves are described by the classical solution of the Korteweg-de Vries equation (Korteweg \& De Vries (1895)). As the amplitude increases the waves ultimately reach a limiting configuration with a stagnation point and a $120^{\circ}$ angle at the crests. We present new simple numerical methods to compute solitary waves of arbitrary amplitude. The basic idea is to represent the solution as an appropriate expansion and to find the unknown coefficients by collocation. These methods are generalisations of similar methods developed previously for periodic waves (see for example Vanden-Broeck (1986)). New numerical results are presented.

The second problem is the classical free surface flow generated by a disturbance moving at a constant velocity $U$. The disturbance can be a submerged object (e.g. a submarine), a floating object (e.g. a ship) or simply a distribution of pressure with a compact support. Many properties of such flows are qualitatively independent of the type of the disturbance. Here we assume that the disturbance is a point vortex of strength $\Gamma_{R}$ moving at the constant velocity $U$. We also restrict our attention to supercritical flows. These simple choices enable us to compute very accurate solutions. We take a frame of reference moving with the point vortex and we seek again steady solutions. Numerical approximations to this problem were previously computed by Elcrat \& Miller (2006). Our computations are in agreement 
with their results. Furthermore, new solutions are obtained by considering the case of an anti-clockwise vortex $\left(\Gamma_{R}<0\right)$. The related problem of free surface waves induced by a point vortex with both positive and negative circulation for subcritical flows in infinite depth is discussed by Forbes (1985).

The connection between the two problems presented in this paper is as follows. When $\Gamma_{R}=0$, a trivial solution of the second problem is a uniform stream characterised by a constant velocity $U$ and a flat free surface. However another solution when $\Gamma_{R}=0$ is a solitary wave (i.e. a solution of the first problem). We can then expect that some of the solutions for $\Gamma_{R}$ small will be close to a uniform stream while others will be close to a solitary wave. This is confirmed by our calculations. Similar results were found before for other types of disturbances (for example, see Dias \& Vanden-Broeck (1989)). Solutions for arbitrary values of $\Gamma_{R}$ are calculated by an extension of the series truncation method used for the first problem. In addition, it is shown that some of the solutions are perturbations of the gravity free solutions previously obtained by Gurevich (1963).

The paper is organised as follows. The first problem is formulated in Section 2. The corresponding numerical results are presented in Section 3. The second problem is studied in Section 4. Concluding remarks are presented in Section 5.

\section{Formulation}

Consider a two-dimensional, inviscid, irrotational and incompressible fluid. The flow is bounded below by a horizontal fixed bottom and above by a free surface, with an undisturbed depth $H$. We assume that a solitary gravity wave of amplitude $P$ travels with a constant velocity $U$. We choose a frame of reference moving with the wave so that the flow is steady. In this frame of reference, the velocity far away from the crest is equal to the velocity $U$ of the wave. We introduce cartesian coordinates with the origin on the bottom. The only body force acting on the fluid is the gravity $g$ which is acting in the negative $y$-direction. We assume that the wave is symmetric about the $y$-axis. The level $y=H$ corresponds to the undisturbed depth. The flow configuration is shown in Fig. 1.

We take $H$ to be the typical length scale and $U$ the typical velocity scale. When non-dimensionalized in this fashion, the undisturbed level is at $y=1$. We take $y=1+\eta(x)$ as the surface displacement of the free surface. The ratio of the amplitude of the wave and the undisturbed depth of the water,

$$
\alpha=P / H
$$

is the dimensionless amplitude.

We denote by $u$ and $v$ the horizontal and vertical components of the velocity and we define the velocity potential and streamfunction by

$$
\begin{gathered}
u=\frac{\partial \phi}{\partial x}=\frac{\partial \psi}{\partial y} \\
v=\frac{\partial \phi}{\partial y}=-\frac{\partial \psi}{\partial x} .
\end{gathered}
$$

Without loss of generality we choose $\psi=0$ on the free surface and $\phi=0$ at the crest of the wave. It follows that $\psi=-1$ on the bottom.

The complete system of equations can be written in terms of $\phi$ as 


$$
\begin{aligned}
\phi_{x x}+\phi_{y y}=0 & & 0<y<1+\eta(x) \\
\phi_{y}=0 & & \text { on } y=0 \\
\phi_{x} \eta_{x}-\phi_{y}=0 & & \text { on } y=1+\eta(x) \\
\frac{1}{2} F^{2}\left(\phi_{x}^{2}+\phi_{y}^{2}\right)+\eta=\frac{F^{2}}{2} & & \text { on } y=1+\eta(x) .
\end{aligned}
$$

where $F$ is the Froude number defined by

$$
F=\frac{U}{\sqrt{g H}} .
$$

Here (2.4) is Laplace equation, (2.5) and (2.6) are the kinematic boundary conditions on the bottom and on the free surface and (2.7) is the dynamic boundary condition on the free surface.

There exists two basic solutions to this system of equations. The first is the trivial solution, a uniform stream defined by

$$
\phi=x \quad \eta=0 \quad F \text { arbitrary. }
$$

The second is the solitary wave solutions, discussed in the following section.

\section{Solitary wave}

We shall reformulate the equations of Section 2 in terms of analytic functions. We introduce the complex potential $f=\phi+i \psi$ and the complex velocity $\xi=u-i v$. It follows from Section 2 that $\xi$ is an analytic function of $f$. The flow domain is now the strip $-1<\psi<0$ in the $f$-plane.

Following Lenau (1966) we map our system from the strip $-1<\psi<0$ in the $f$-plane to a unit semi-circle in the $t$-plane via the function

$$
f=\frac{2}{\pi} \log \frac{1+t}{1-t}-i
$$

We then use the method of images to reflect the flow across the line $-1 \leqslant t \leqslant 1$. The complex $t$-plane is illustrated in Fig. 2. The free surface is mapped on the upper semi-circle $A C B$. We can then represent it by

$$
t=e^{i \sigma} \quad \text { where } \quad 0<\sigma<\pi .
$$

Next we differentiate the free surface condition (2.7) with respect to $\sigma$. This yields

$$
F^{2}\left(u(\sigma) u_{\sigma}(\sigma)+v(\sigma) v_{\sigma}(\sigma)\right)-\frac{v(\sigma)}{u(\sigma)^{2}+v(\sigma)^{2}}\left(\frac{2}{\pi} \frac{1}{\sin \sigma}\right)=0,
$$

where $u(\sigma)$ and $v(\sigma)$ and the values of $u$ and $v$ on the free surface.

We seek $\xi$ as an analytic function of $t$. The basic idea of our numerical method is to represent $\xi$ as a Taylor expansion in powers of $t$. For this series to be convergent, all leading singularities in $|t| \leqslant 1$ must be removed. The function $|\xi|$ has singularities at $t= \pm 1$. This comes from the fact that

$$
\xi \approx 1+A e^{-\pi \lambda|\phi|} \quad \text { as } \quad|\phi| \rightarrow \infty
$$


where $\lambda$ is the smallest positive root of

$$
\pi \lambda-\frac{\tan \pi \lambda}{F^{2}}=0
$$

(see Hunter \& Vanden-Broeck (1983) for details). It then follows from (3.1) that

$$
\xi \sim(t \mp 1)^{2 \lambda} \text { as } \quad t \rightarrow \pm 1 .
$$

We write an expansion for $\xi$ where the singularity (3.6) is removed. One such choice is

$$
\xi=\exp \left\{A\left(1-t^{2}\right)^{2 \lambda}+\sum_{k=1}^{\infty} a_{k}\left(t^{2 k}-1\right)\right\},
$$

where $A$ and the coefficients $a_{k}$ have to be determined. This is achieved numerically.

We truncate the series (3.7) after $N$ terms, leaving us with $N+3$ unknowns: $a_{1}, a_{2}, \ldots, a_{N}, \lambda, A$ and $F$. We satisfy the free surface boundary condition at $N+1$ equally spaced mesh points given by

$$
\sigma_{I}=\frac{\pi}{2(N+1)}\left(I-\frac{1}{2}\right) \quad I=1,2, \cdots, N+1,
$$

as well as equation (3.5). We require one more equation so that our system is not underdetermined. We could choose to fix a value of $\alpha$ where $\alpha$ is defined by (2.1). However a more convenient choice is to fix $\omega$ where $\omega$ is defined by

$$
\omega=1-F^{2} u_{c}^{2} .
$$

Here $u_{c}$ is the value of $u$ at the crest (i.e. at $\sigma=\pi / 2$ ). The motivation for using (3.9) is that it is a priori known that $0 \leqslant \omega \leqslant 1$ for all possible waves. This can easily be checked by noting that $\omega \rightarrow 0$ as the amplitude tends to zero (since $u_{c} \rightarrow 1$ and $F \rightarrow 1$ ) and $\omega \rightarrow 1$ as the limiting configuration is approached (since $u_{c} \rightarrow 0$ ). The system of $N+3$ nonlinear equations with $N+3$ unknowns is solved by Newton's method. The profile of the wave is then obtained in parametric form by integrating numerically the identity

$$
x_{\phi}+i y_{\phi}=\frac{1}{u-i v},
$$

which can be rewritten by using (3.1) and the chain rule as

$$
x_{\sigma}+i y_{\sigma}=-\frac{2}{\pi \sin \sigma} \frac{1}{u-i v} .
$$

Solitary waves of very small amplitude are described asymptotically by the classical solution of the Korteweg-de Vries equation. This solution can be written in the form

$$
\eta(x)=2(F-1) \operatorname{sech}^{2}\left[\left(\frac{6(F-1)}{4}\right)^{1 / 2} x\right] .
$$

To check our numerical procedure, we compare in Fig. 3 our numerical solutions with the profile (3.12). As expected a good agreement is found for $\omega$ small. For values of $\omega$ not too close to 1 , we found that the code converges quickly as $N$ increases. In particular the coefficients $a_{n}$ decrease rapidly as $n$ increases. For example, for $\omega=0.8, a_{1} \approx 1.5 \times 10^{-1}, a_{10} \approx-3.6 \times 10^{-3}, a_{20} \approx-1.5 \times 10^{-4}, a_{50} \approx 4.7 \times 10^{-7}$. 
However the convergence deteriorates as the wave of maximum amplitude is approached (i.e. as $\omega \rightarrow 1$ ). This is illustrated in Fig. 4 where we present values $F$ versus $\omega$ computed by using (3.7) with $N=100$ and $N=300$. We also show in the Figure the accurate values $F$ versus $\omega$ obtained by a different method described below. The results show that larger and larger values of $N$ are needed in the truncated version of (3.7) as $\omega \rightarrow 1$. In particular the code does not converge for $\omega=1$. This is due to the fact

$$
\xi \sim f^{1 / 3} \quad \text { as } \quad f \rightarrow 0
$$

for the limiting configuration. It then follows from (3.1) that

$$
\xi \sim(t-i)^{1 / 3} \text { as } t \rightarrow i
$$

This suggests that the highest solitary wave can be computed by representing $\xi$ by the series

$$
\xi=\left[\frac{1}{2}\left(1+t^{2}\right)\right]^{\frac{1}{3}} \exp \left\{A\left(1-t^{2}\right)^{2 \lambda}+\sum_{k=1}^{\infty} a_{k}\left(t^{2 k}-1\right)\right\} .
$$

We then proceed as we did with (3.7). However we do not impose the equation (3.9) because this equation is automatically satisfied when $\omega=1$. Therefore we replace $N+1$ by $N+2$ in (3.8) so that the number of unknowns is still equal to the number of equations. This code was used by Hunter $\&$ Vanden-Broeck (1983) who computed solutions for $N \leqslant 100$. These calculations were extended in Vanden-Broeck (2010) up to $N=1200$. We present in Table 1 values of $\alpha$ and $F$ up to $\mathrm{N}=7000$. These results illustrate clearly the convergence of the method. Our value for the amplitude with $\mathrm{N}=7000$ is in good agreement with that of Evans \& Ford (1996).

So far we have two codes. The one based on (3.7) works up to moderate values of $\omega$ whereas the one based on (3.15) works for $\omega=1$. A code working for intermediate values of $\omega$ can be derived by using the series representation

$$
\xi=\left[\frac{1+\beta t^{2}}{1+\beta}\right]^{\frac{1}{3}} \exp \left\{A\left(1-t^{2}\right)^{2 \lambda}+\sum_{k=1}^{\infty} a_{k}\left(t^{2 k}-1\right)\right\}
$$

where the parameter $\beta$ is found as part of the solution. The motivation is that (3.16) reduces to (3.7) as $\beta \rightarrow 0$ and to (3.15) for $\beta=1$. Therefore (3.16) combines the advantages of both (3.7) and (3.15). We again proceed as we did with (3.7). We have now the extra unknown $\beta$. Therefore we need to replace $N+1$ by $N+2$ in (3.8) to keep the number of unknowns equal to the number of equations. The idea behind (3.16) goes back to Havelock (1918) who applied it to calculate periodic waves. It was later used by Vanden-Broeck (1986), who computed Havelock's expansion with a much larger number of coefficients. Equation (3.16) was quoted in Vanden-Broeck (2010) but no computations were presented.

Numerical values of $F$ versus $\omega$ obtained by using (3.16) with $N=100$ are shown in Fig. 4 . These values remain unchanged when larger values of $N$ are used. This is to be contrasted with the behaviour observed when using (3.7) (see dotted and broken lines in Fig. 4).

Figure 4 shows that $F$ reaches a maximum at $\omega \approx 0.92$. We found that as the limiting configuration is approached (i.e. as $\omega \rightarrow 1$ ), there exists an infinite sequence of minima and maxima of $F$, a property found numerically by Longuet-Higgins \& Fox (1978) for periodic waves on infinitely deep water. Plots obtained close to $\omega=1$ show that the graph approaches the first minimum at about $\omega \approx 0.996$ (see Fig. $5)$. 


Table 1. Maximum amplitudes vs N
\begin{tabular}{|c|c|c|}
\hline $\mathrm{N}$ & $\alpha$ & $\mathrm{F}$ \\
\hline 100 & 0.83322435 & 1.29091003 \\
\hline 200 & 0.83321702 & 1.29090435 \\
\hline 400 & 0.83320877 & 1.29089796 \\
\hline 800 & 0.83320372 & 1.29089405 \\
\hline 1600 & 0.83320116 & 1.29089206 \\
\hline 3000 & 0.83320005 & 1.29089120 \\
\hline 4800 & 0.83319962 & 1.29089087 \\
\hline 6000 & 0.83319949 & 1.29089077 \\
\hline 7000 & 0.83319942 & 1.29089071 \\
\hline Ford & 0.83319918 & 1.29089053 \\
\hline
\end{tabular}

\section{Free surface flow past a point vortex}

The results in this section provide models for free surface flows caused by a disturbance moving at constant velocity with a frame of reference moving with the disturbance. The mathematical properties of such flows are qualitatively independent of the type of disturbance (see Vanden-Broeck (2010)). For mathematical convenience, we have chosen the disturbance to be a point vortex of strength $\Gamma_{R}$. We also assumed that the flow is supercritical (i.e. that $F>1$ ). There are then no waves and the free surface is flat as $|x| \rightarrow \infty$.

The flow configuration is illustrated in Fig. 1. The flow is again characterised by a uniform stream with constant velocity $U$ and constant depth $H$ as $|x| \rightarrow \infty$. As in Section 2, we take $U$ as the reference velocity and $H$ as the reference length. The dimensionless strength of the vortex is then

$$
\Gamma=\frac{\Gamma_{R}}{U H}
$$

When $\Gamma=0$, the system of equations governing the flow is identical to those in Section 3, given by (2.4) - (2.7). For this system of equations there are two families of solutions. The first is a uniform stream, defined by (2.9). The second is the branch of solitary waves, described in detail in Section 3 . Variations in the strength of the vortex $\Gamma$ should then produce solution branches that can be viewed as perturbations of either the uniform stream or a solitary wave. This is confirmed by the computations and results presented in this section.

\subsection{Exact solution for $g=0$}

We first consider an exact solution of the problem found by Gurevich (1963) when the effects of gravity are ignored (i.e. as $F \rightarrow \infty$ ). We shall then adapt this solution, using ideas from Section 3, to construct a numerical procedure to compute such flows when gravity is included.

As $F \rightarrow \infty$, the dynamic boundary condition (2.7) becomes

$$
u^{2}+v^{2}=1 \quad \text { or } \quad|\xi|=1
$$

As in Section 3, it is advantageous to map the flow domain to a unit semi-circle in the $t$-plane. However, the domain in the $f$-plane is no longer the infinite strip $-1<\psi<0$. This is due to the 
logarithmic singularity caused by the point vortex. Gurevich (1963) showed that the flow of Fig. 1 can still be mapped onto the unit circle in the $t$-plane of Fig. 2 by the transformation

$$
f=\frac{2}{\pi} \log \frac{1+t}{1-t}-i+\frac{i \Gamma}{2 \pi} \log \frac{(t-i \gamma)\left(t+i \gamma^{-1}\right)}{(t+i \gamma)\left(t-i \gamma^{-1}\right)} .
$$

Using Chaplygin's singular point method (for details on this method, see Gurevich (1966)), Gurevich then derived the following exact solution for the gravity free problem

$$
\xi=\frac{\left(t^{2}-\delta^{2}\right)\left(t^{2} \gamma^{2}+1\right)}{\left(1-\delta^{2} t^{2}\right)\left(t^{2}+\gamma^{2}\right)}
$$

Here, $\chi$ is defined by

$$
\chi=\frac{2\left(\gamma^{-2}+\gamma^{2}\right)+\Gamma\left(\gamma^{-1}-\gamma\right)}{4-\Gamma\left(\gamma^{-1}-\gamma\right)},
$$

and $\delta$ is a stagnation point in the $t$-plane defined by

$$
\begin{array}{ll}
\delta=\frac{i}{\sqrt{\sqrt{\chi^{2}-1}+\chi}} & \text { for } \chi>0, \\
\delta=\frac{1}{\sqrt{\sqrt{\chi^{2}-1}-\chi}} & \text { for } \chi<0,
\end{array}
$$

For $\Gamma>0$ the free surface is in the form of an elevation wave whereas it is in the form of a depression wave for $\Gamma<0$. It is of interest to note that Shaw (1972) demonstrated that the solution given by (4.4) does not describe the flow if $|\chi|<1$, since then the stagnation point $\delta$ is on the free surface. This occurs if $\Gamma<0$ and

$$
\gamma>\left((\Gamma / 2)^{2}+1\right)^{1 / 2}+\Gamma / 2=\gamma_{0} .
$$

Shaw corrected Gurevich's solution for this case, noting that the stagnation point $\delta$ is no longer in the flow, and that the solution is instead characterized by a bifurcation point on the free surface. The solution can be written as

$$
\xi=\frac{\left(t^{2} \gamma^{2}+1\right)}{\left(t^{2}+\gamma^{2}\right)}
$$

This solution is valid for $\gamma^{*}<\gamma<1$, where $\gamma^{*}$ is dependant on $\Gamma$. For values of $\gamma<\gamma^{*}$, the free surface of Shaw's solution (4.9) self intersects. Hence, there is yet to be found an exact weightless solution for values of $\gamma \in\left(\gamma_{0}, \gamma^{*}\right)$ when $\Gamma<0$. Typical free surface profiles are shown in Fig. 6 (for further details, see Shaw (1972)).

We conclude this subsection by discussing the possible limiting configurations of the free surface flows with $g=0$. As $\gamma \rightarrow 1$, the flow tends to a flat free streamline with a circle of radius $|\Gamma| / 2 \pi$ balanced on top. Figure 7 demonstrates the approach to the limiting configuration for $\Gamma>0$ and $\Gamma<0$. The limiting configuration given for $\Gamma>0$ is not a solution since there exists a stagnation point on the free surface. However, the limiting configuration for $\Gamma<0$ is a valid solution, since instead of a stagnation point there is a bifurcation point on the free surface. 
4.2 Numerical solutions when $g \neq 0$

Next, we consider the flow described in Section 4.1, but with $g \neq 0$. This causes a change in the dynamic boundary condition on the free surface, which is now of the form (2.7). However, the kinematic boundary conditions (2.5) and (2.6) on the wall and on the free surface remain unchanged. Therefore, the condition that $\psi$ is constant on the wall and on the free surface is still true, and hence the complex potential of the flow is given by (4.3). However, the solution (4.4) no longer satisfies the dynamic boundary condition.

To find the solution with this new boundary condition on the free surface, we adapt the exact solution using ideas from Section 3. The flow has the asymptotic behaviour (3.6) at $t= \pm 1$. However, the flow now has singularities associated with the point vortex at $t=i \gamma$ and the stagnation point $\delta$ in the flow domain.

Hence, we represent $\xi$ by the expansion

$$
\xi=\frac{\left(t^{2}-\delta^{2}\right)\left(\gamma^{2} t^{2}+1\right)}{\left(1-t^{2} \delta^{2}\right)\left(t^{2}+\gamma^{2}\right)} \exp \left\{A\left(1-t^{2}\right)^{2 \lambda}+\sum_{k=1}^{\infty} a_{k}\left(t^{2 k}-1\right)\right\},
$$

where $A$ and the coefficients $a_{k}$ have to be determined. For simplicity, we only consider values of $\Gamma$ and $\gamma$ such that $\chi>1$. This ensures that the expansion, with $\delta$ defined by (4.6), always has the correct singular behaviour of the flow for $|t| \leqslant 1$.

As $F \rightarrow \infty$, the numerical computations described below show that $A \rightarrow 0$ and $a_{k} \rightarrow 0$ for $k=1,2, \ldots$ Therefore (4.10) reduces to (4.4) as $F \rightarrow \infty$. Furthermore (4.6) and (4.7) imply that $\delta^{2} \rightarrow-\gamma^{2}$ as $\Gamma \rightarrow 0$, so that (4.10) reduces to (3.7) when $\Gamma=0$.

As shown by Elcrat \& Miller (2006), one of the limiting configurations of the flows with $\Gamma \neq 0$ and $F \neq \infty$ is still characterised by a stagnation point with a $120^{\circ}$ angle. Such a solution cannot be computed by using (4.10). However this can be achieved by following the discussion of Section 3 and representing $\xi$ by the expansion

$$
\xi=\left[\frac{1+t^{2}}{2}\right]^{\frac{1}{3}} \frac{\left(t^{2}-\delta^{2}\right)\left(\gamma^{2} t^{2}+1\right)}{\left(1-t^{2} \delta^{2}\right)\left(t^{2}+\gamma^{2}\right)} \exp \left\{A\left(1-t^{2}\right)^{2 \lambda}+\sum_{k=1}^{\infty} a_{k}\left(t^{2 k}-1\right)\right\}
$$

As $\Gamma \rightarrow 0,(4.11)$ reduces to (3.15) since $\delta^{2} \rightarrow-\gamma^{2}$.

Following the derivation of (3.16), we can combine the advantages of (4.10) and (4.11) by writing

$$
\xi=\left[\frac{1+\beta t^{2}}{1+\beta}\right]^{\frac{1}{3}} \frac{\left(t^{2}-\delta^{2}\right)\left(\gamma^{2} t^{2}+1\right)}{\left(1-t^{2} \delta^{2}\right)\left(t^{2}+\gamma^{2}\right)} \exp \left\{A\left(1-t^{2}\right)^{2 \lambda}+\sum_{k=1}^{\infty} a_{k}\left(t^{2 k}-1\right)\right\}
$$

Since the limiting configuration is defined by a stagnation point at the crest, using (2.7) we find that the maximum possible amplitude achieved for a fixed $F$ is given by

$$
\alpha_{\max }=F^{2} / 2
$$

Values of $\alpha$ versus $1 / F$ for various values of the parameters are shown in Fig. 8. The broken curve corresponds to the limiting configuration defined by (4.13). Typical free surface profiles for solutions on branches $(a)$ and $(b)$ from Fig. 8 are shown in Figs 9 - 10.

We found that a solitary gravity wave containing a clockwise vortex $(\Gamma>0)$ is steeper and faster than an unperturbed solitary wave of the same amplitude. Furthermore, a higher maximum amplitude 
is achieved. All such branches lie in the space bounded by the axis, the solitary wave branch (e) and the broken curve in Fig. 8. The $\alpha$-axis $(1 / F=0)$ corresponds to the weightless solutions given in Section 4.1, while the $1 / F$-axis $(\alpha=0)$ corresponds to the uniform stream solution (2.9). Hence, the section of the solution branches close to the $\alpha$-axis are best seen as perturbations of the exact solution (4.4) with small values of gravity $g$. Meanwhile, solutions close to the $1 / F$-axis are perturbations of the uniform stream with a point vortex, and the final section of the solution branches are perturbations of the solitary wave solutions from Section 3 with a point vortex. Close to the maximum amplitude solutions (dashed line in Fig. 8), we see the non-monotonic behaviour between $\alpha$ and $F$ previously shown for the unperturbed solitary wave solutions (Fig. 5).

For solitary waves containing an anti-clockwise vortex $(\Gamma<0)$, we found that for a fixed amplitude the perturbed wave was slower and more shallow. A lower maximum amplitude is achieved. As we follow the branch for decreasing amplitude, the wave starts to form a depression on the line of symmetry. Once this happens, the value $\alpha$ is no longer the highest point on the wave. We denote by $Y$ the maximum displacement from the line of undisturbed depth. We found that the solution with a depression in the middle has a very similar value of $F$ as the undisturbed wave with the same value of $Y$. We suspect that this perturbed solution then approaches a limiting configuration of two pointed crests with a $120^{\circ}$. However, the position of this singularity is no longer known in the $t$-plane, since the crest is no longer at $x=0$, and hence we cannot use the expansion (4.11) to compute this limiting configuration. Typical profiles are shown in Fig. 11.

\section{Conclusion}

We have developed new and rapidly converging numerical schemes to compute highly accurate solutions for solitary gravity waves of arbitrary amplitude. Adapting the solution of weightless flow past a point vortex, a solution first derived by Gurevich, we constructed a numerical scheme which allows us to perturb the solitary wave solutions. Results were obtained for both a clockwise and anti-clockwise vortex. We found that a clockwise vortex increased the maximum possible amplitude of the solution, while an anti-clockwise vortex decreased this value.

\section{Funding}

This work was supported by the Engineering and Physical Sciences Research Council [EP/M507970/1 to A.D., EP/N018559/1, EP/J019569/1 to J.-M V.-B.].

\section{REFERENCES}

Dias, F. \& Vanden-Broeck, J.-M. (1989) Open channel flows with submerged obstructions. Journal of Fluid Mechanics, 206, 155-170.

Elcrat, A. R. \& Miller, K. G. (2006) Free surface waves in equilibrium with a vortex. European Journal of Mechanics-B/Fluids, 25(2), 255-266.

Evans, W. \& Ford, M. (1996) An exact integral equation for solitary waves (with new numerical results for some'internal'properties). Proceedings of the Royal Society of London A: Mathematical, Physical and Engineering Sciences, 452(1945), 373-390.

Forbes, L. (1985) On the effects of non-linearity in free-surface flow about a submerged point vortex. Journal of Engineering Mathematics, 19(2), 139-155.

Gurevich, M. I. (1963) Vortex near a free surface. Journal of Applied Mathematics and Mechanics, 27(5), 13701376.

Gurevich, M. I. (1966) Theory of jets in ideal fluids. Academic Press. 
Havelock, T. (1918) Periodic irrotational waves of finite height. Proceedings of the Royal Society of London. Series A, 95(665), 38-51.

Hunter, J. \& Vanden-Broeck, J.-M. (1983) Accurate computations for steep solitary waves. Journal of Fluid Mechanics, 136, 63-71.

Korteweg, D. J. \& De Vries, G. (1895) Xli. on the change of form of long waves advancing in a rectangular canal, and on a new type of long stationary waves. The London, Edinburgh, and Dublin Philosophical Magazine and Journal of Science, 39(240), 422-443.

Lenau, C. W. (1966) The solitary wave of maximum amplitude. Journal of Fluid Mechanics, 26(02), 309-320.

Longuet-Higgins, M. \& Fox, M. (1978) Theory of the almost-highest wave. Part 2. Matching and analytic extension. Journal of Fluid Mechanics, 85(04), 769-786.

Schwartz, L. \& Fenton, J. (1982) Strongly nonlinear waves. Annual review of fluid mechanics, 14(1), 39-60.

Shaw, S. J. (1972) A note on the potential vortex in a wall jet. Quarterly of Applied Mathematics, 30, 351-356.

Vanden-Broeck, J.-M. (1986) Steep gravity waves: Havelocks method revisited. Physics of Fluids, 29(9), 30843085.

Vanden-Broeck, J.-M. (2010) Gravity-capillary free-surface flows. Cambridge University Press.

\section{Figures}

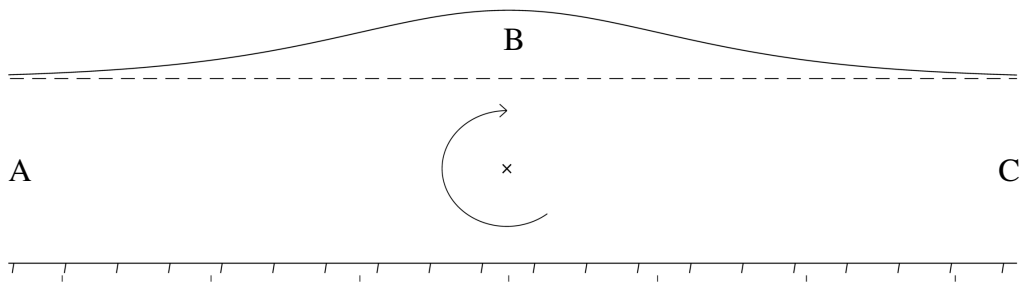

FIG. 1. Sketch of the flow in the $z$-plane. 


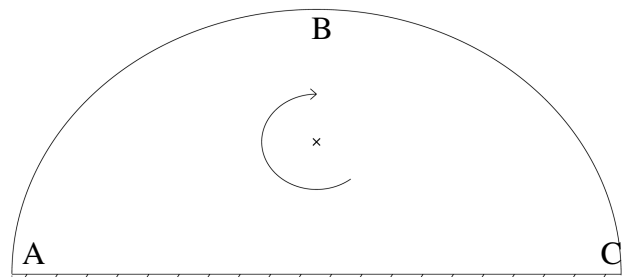

FiG. 2. Sketch of the flow in the $t$-plane. The cross denotes the point vortex (it is not present in the first problem). The portion of the sketch below $A B$ is the image of the flow into the bottom. 

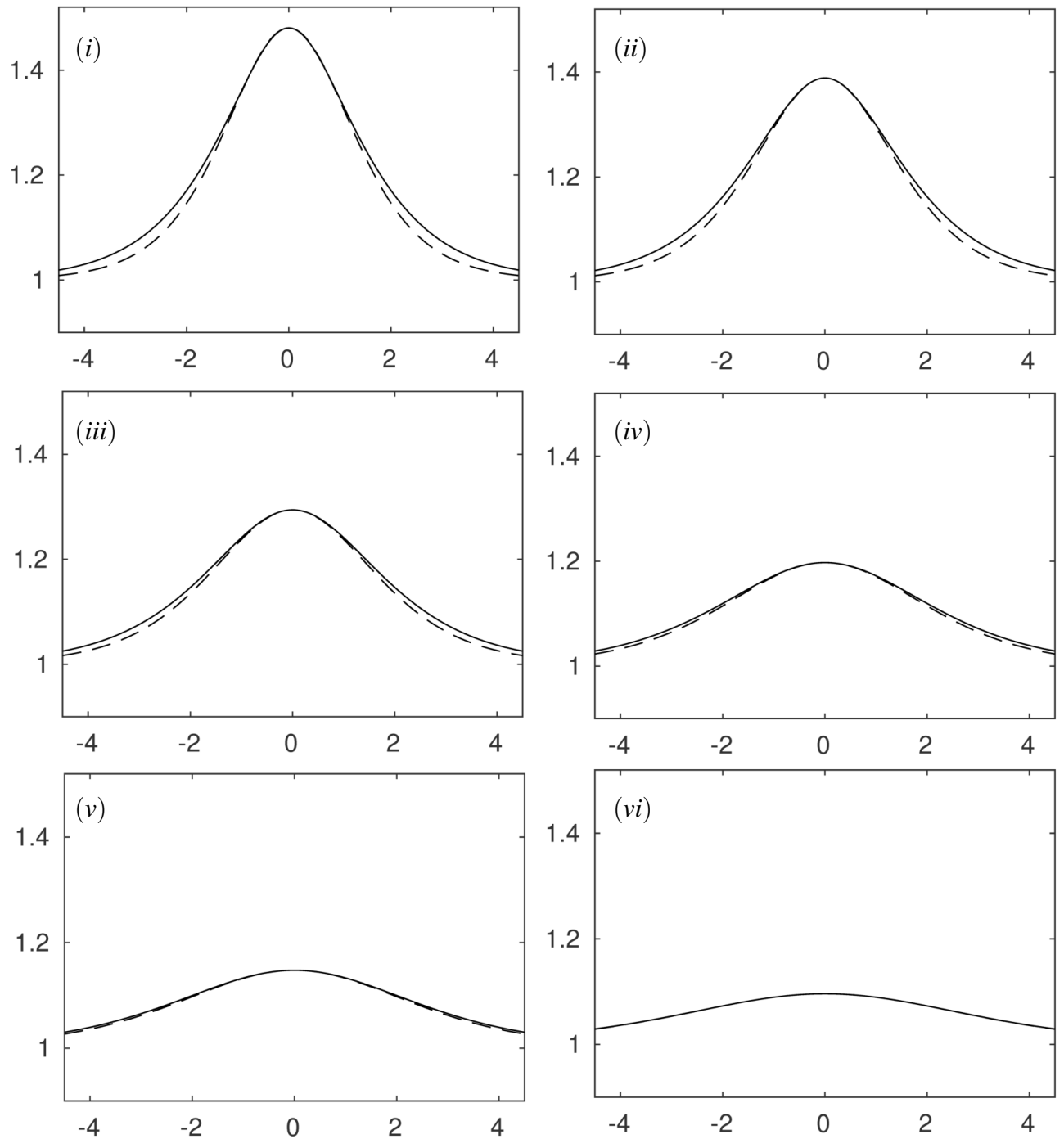

FIG. 3. Plots of the solitary wave solutions computed in Section 3 (solid curves), compared against the KdV solution (dashed curves) given by (3.12) for various values of $\omega$. The solutions correspond to: (i) $\omega=0.5$, (ii) $\omega=0.4$, (iii) $\omega=0.3,($ iv $) \omega=0.2$, (v) $\omega=0.15,(v i) \omega=0.1$ 


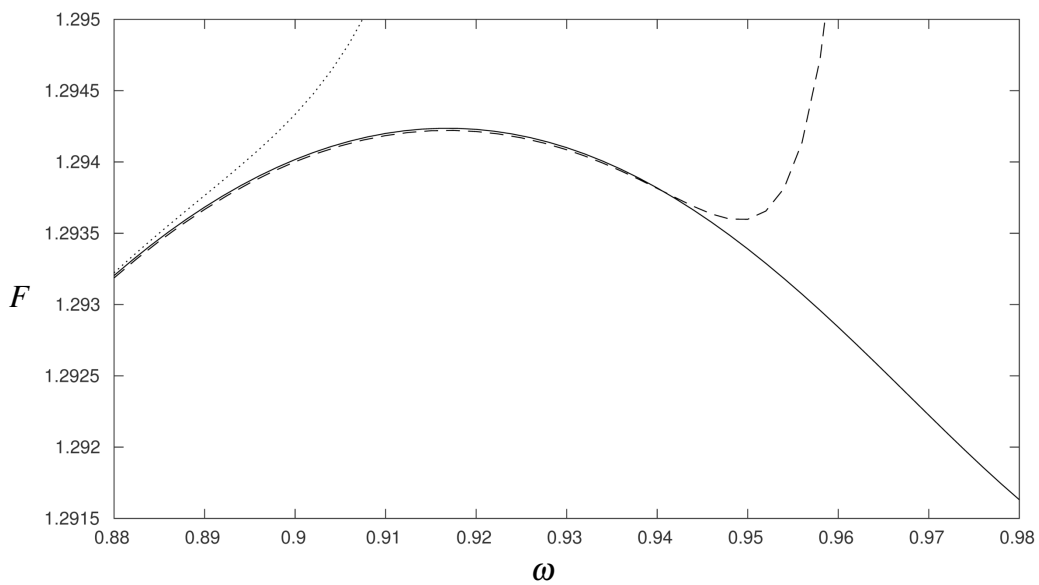

FIG. 4. Plot of F versus $\omega$ for larger values of $\omega$. The dotted and dashed line are computed by using the series $(3.7)$ for $N=100$ and $N=300$ respectively. The solid line is computed using the series (3.16) with $N=100$.

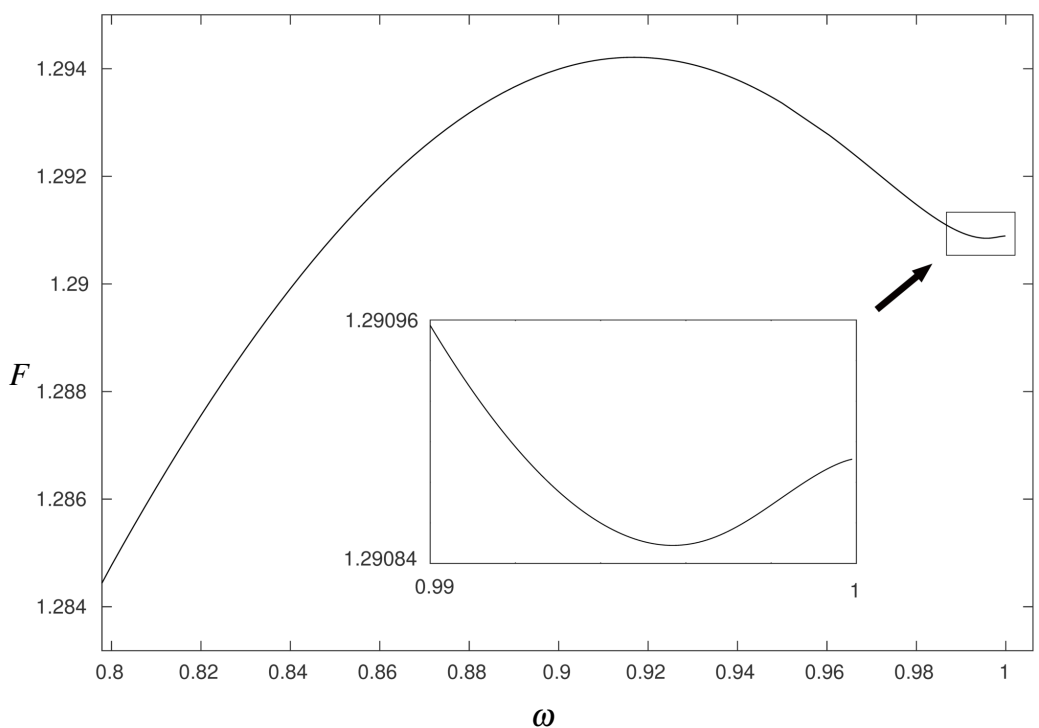

FIG. 5. Plot of F versus $\omega$ computed with $N=2000$. The first minimum is shown to occur at $\omega \approx 0.996$. 

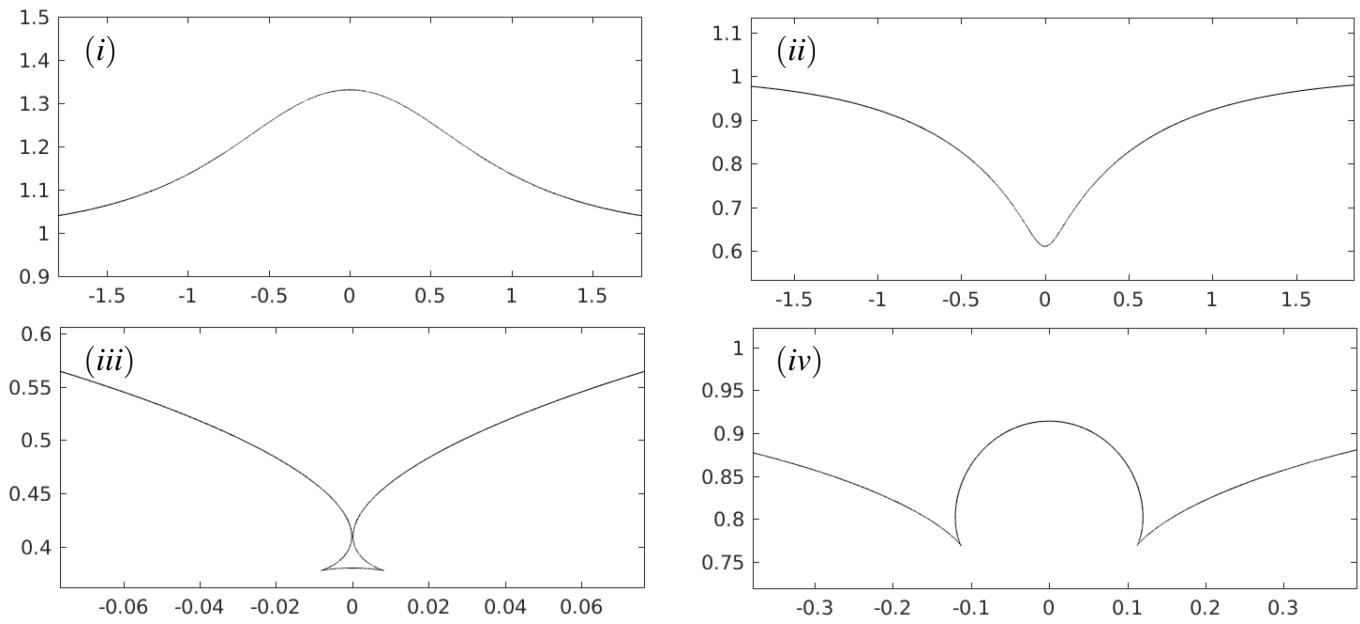

FIG. 6. The solution of the problem formulated in Section 4.1 for a variety of parameter values. Plots $(i)$ and $(i i)$ are solutions of the form (4.4) with parameter values $(i) \Gamma=1, \gamma=0.5$ and (ii) $\Gamma=-1, \gamma=0.5$. Plots (iii) and (iv) are given by (4.9). Both are with $\Gamma=-1$. The values of $\gamma$ are (iii) $\gamma=0.6535 \approx \gamma^{*}$ and (iv) $\gamma=0.9$. In plot (iii) we can see that the free surface touches itself at $x=0$. If we decrease $\gamma$ further the free surface self intersects. We can see clearly in $(i v)$ the location of the bifurcation points on the free surface.
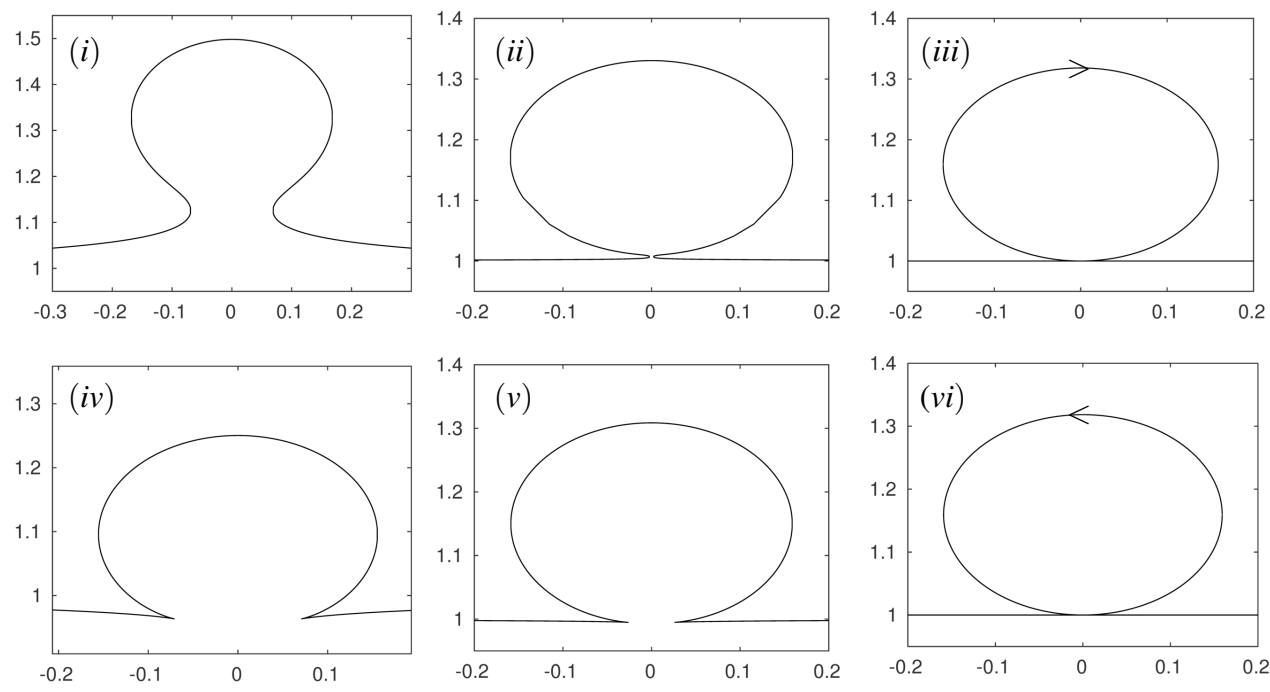

FIG. 7. The top row consists of plots of the solution (4.4) as $\gamma \rightarrow 1$, while the bottom row shows the solution (4.9) as $\gamma \rightarrow 1$. For the top row,$\Gamma=1$, (i) $\gamma=0.999$, (ii) $\gamma=0.999999$, (iii) $\gamma=1$. For the bottom row, $\Gamma=-1,(i v) \gamma=0.99,(v) \gamma=0.999$, (vi) $\gamma=1$. 


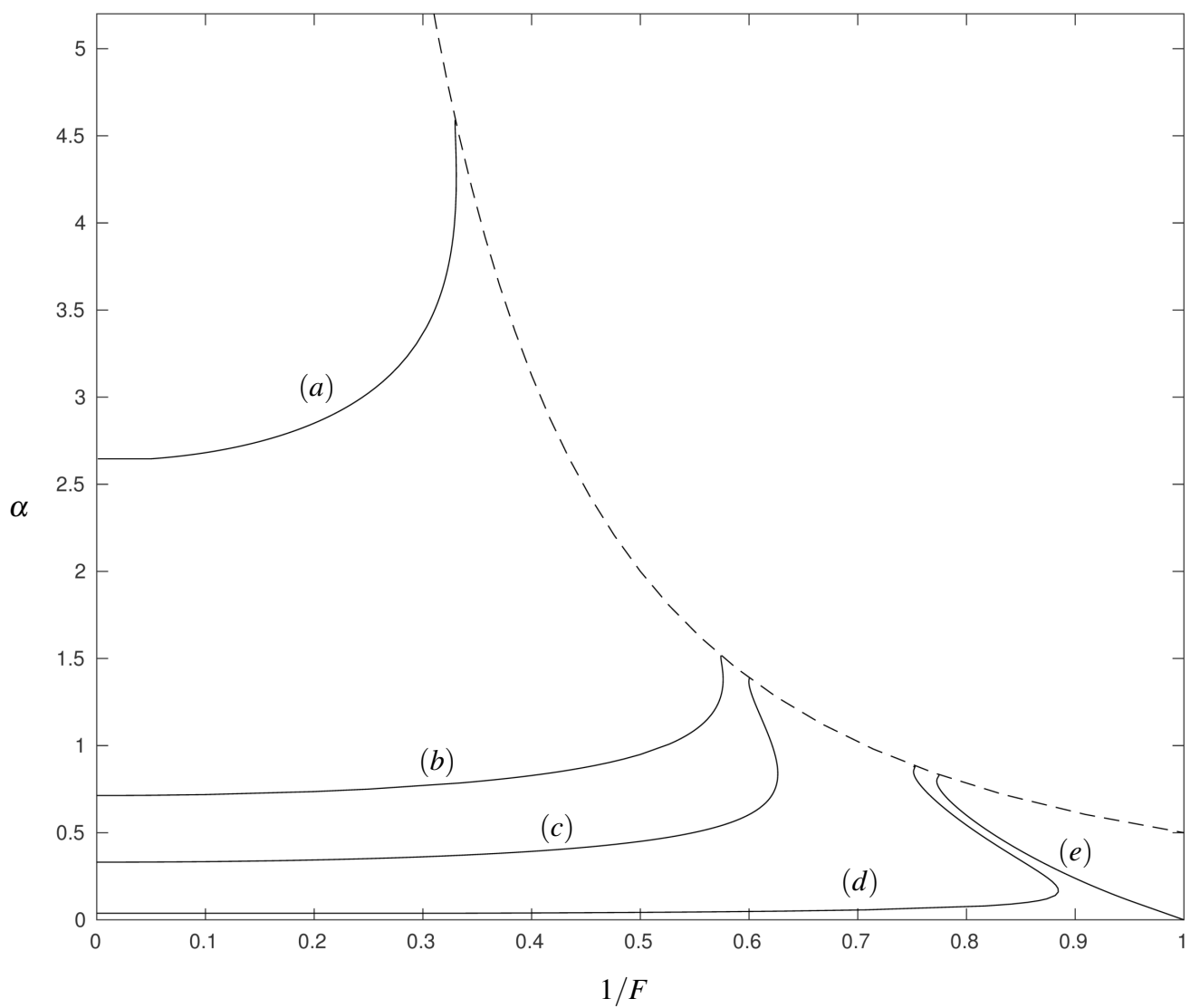

FIG. 8. A plot of the solution branches in the $(1 / F, \alpha)$ plane. The solution branch (a) has parameter values $\gamma=0.95, \Gamma=5$, (b) $\gamma=0.95, \Gamma=1$ (c) $\gamma=0.5, \Gamma=1$, and (d) $\gamma=0.5, \Gamma=0.1$. The branch (e) is the solution branch of unperturbed gravity waves, computed in Section 3. The dashed line is the plot of $\alpha_{\max }$ given by (4.13), at which the solution branches terminate, since the amplitude cannot exceed this critical value. 

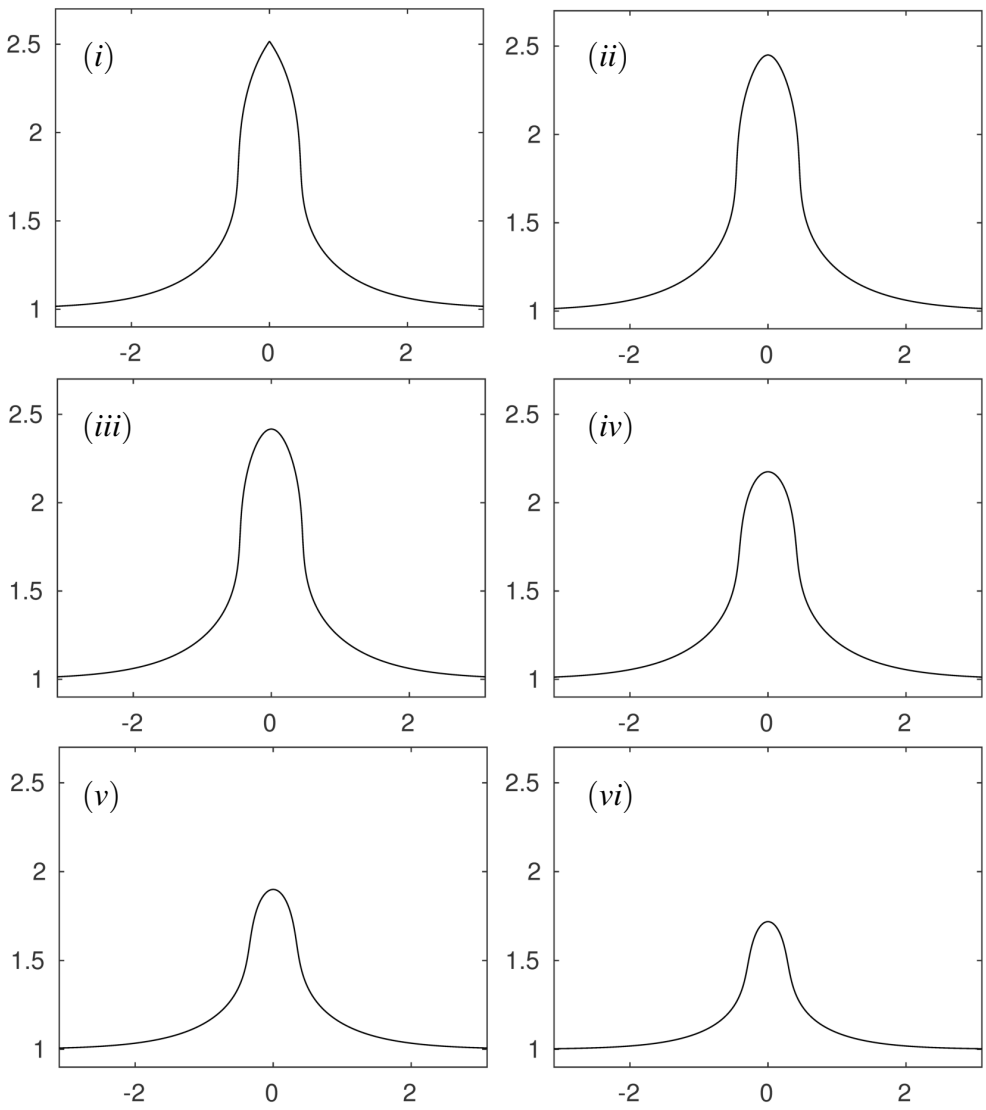

FIG. 9. Profiles of solutions from branch (b) in Fig. 8 (parameter values $\Gamma=1$ and $\gamma=0.95$ ). The maximum amplitude solution (i) has values $\alpha=1.52, F=1.75$. The other solutions have the values (ii) $\alpha=1.49, F=1.74$, (iii) $\alpha=1.41, F=1.73$, (iv) $\alpha=1.17, F=1.77,(v) \alpha=0.90, F=2.13$, and $(v i) \alpha=0.71, F=10$ 

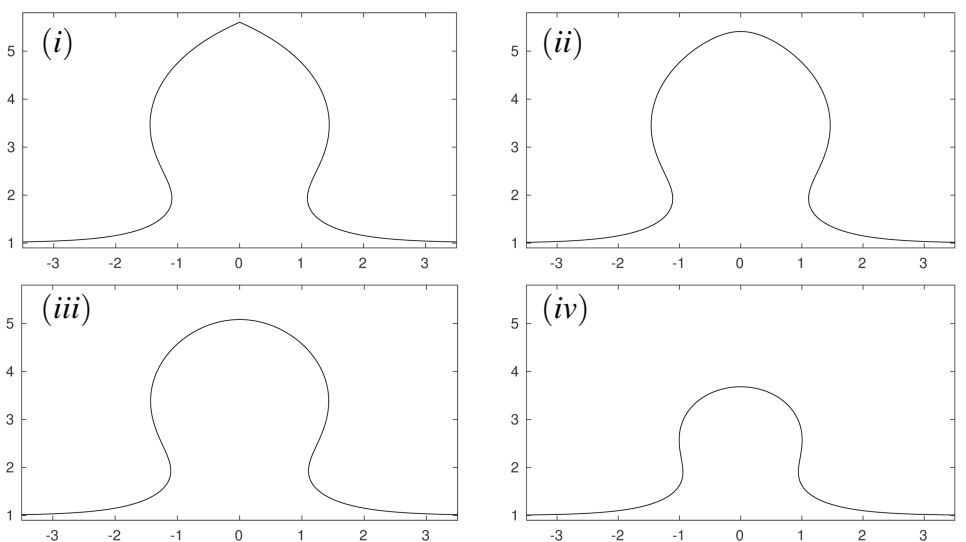

FIG. 10. Profiles of solutions from branch (a) in Fig. 8 (parameter values $\Gamma=5$ and $\gamma=0.95$ ). The maximum amplitude solution (i) has the values $\alpha=4.60, F=3.03$. The other solutions have the values (ii) $\alpha=4.41, F=3.02$, (iii) $\alpha=4.08, F=3.03$ and (iv) $\alpha=2.68, F=10$.
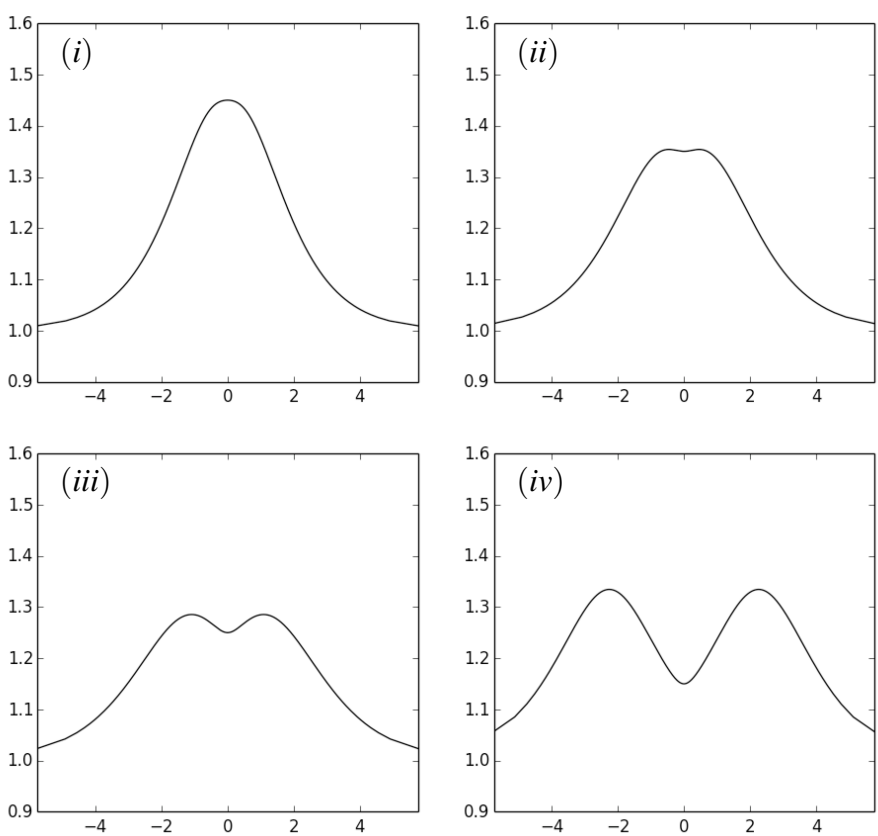

FIG. 11. Profiles for the parameter values $\Gamma=-0.1, \gamma=0.5$ and $(i) \alpha=0.45$, (ii) $\alpha=0.35$, (iii) $\alpha=0.25$ and (iv) $\alpha=0.15$. Notice that the maximum height achieved is no longer given by $\alpha$. 